\title{
OS 4PS DO COMPOSTO DE MARKETING NA LITERATURA DE CIÊNCIA DA INFORMAÇÃO
}

\section{THE FOUR PS OF MARKETING MIX IN INFORMATION SCIENCE LITERATURE}

\author{
Sueli Angelica do AMARAL*
}

RESUMO

\begin{abstract}
Analisa 60 documentos sobre os 4 Ps do composto de marketing em unidades de informação da literatura de Ciência da Informação no período de 1975 a 1995, para conhecer quantos e quais autores escreveram sobre o tema, qual o pioneiro, quem foi o pioneiro a propor adição de Ps. Apresenta a cronologia dos documentos estudados e discute as 4Ps do composto de marketing. Propõe a adoção da mais completa proposta analisada como forma de garantir o futuro das unidades de informação.
\end{abstract}

Palavras-chave: composto de marketing, marketing mix, composto mercadológico, unidades de informação.

\section{ABSTRACT}

The paper analyzes 60 documents about the 4Ps of marketing mix in libraries in Information Science literature from 1975 to 1995, in order to know the writers and how many have written about the topic. It also asks such questions as: Who was the pioneer? Who was the pioneer to add Ps? It shows a chronology of the reviewed documents and it comments on the 4Ps, and suggests the adoption of the most completed addition proposal presented in order to guarantee library future.

Key Words: marketing mix, information agencies

\section{INTRODUÇÃO}

Os estudiosos e interessados em marketing, na área de informação, certamente se deparam com algumas dificuldades ao realizar pesquisas bibliográficas em tópicos mais específicos sobre o tema.

Antes de tudo, é preciso considerar o pouco tempo da transferência dos conceitos mercadológicos para o setor que não visa ao lucro, no qual se inserem as unidades de informação, sejam elas bibliotecas tradicionais ou virtuais; setores, divisões, centros, sistemas de documentação e/ou informação ou qualquer outro tipo de organização atuante para captar, selecionar, organizar, estruturar e processar adequadamente, gerar, disseminar e recuperar informação, não importa em que tipo de suporte ou qual seja a extensão dessas atividades. Somente em 1969, portanto há pouco mais de trinta anos, os autores Kotler \& Levy (1969) admitiram a possibilidade de aplicação das técnicas mercadológicas pelas instituições do setor que não visa ao lucro, uma

\footnotetext{
(") Professora Adjunta do Departamento de Ciência da Informação e Documentação da Universidade de Brasília)
} 
vez que, até então, essas técnicas só eram utilizadas pelas organizações do setor lucrativo. Ainda que o assunto estimulasse o considerável aumento de adeptos à proposta e à literatura mundial no âmbito de Ciência da Informação sobre o tema tenha se desenvolvido plenamente, durante esse curto período de tempo decorrido após a inovação proposta em 1969 pelos autores mencionados, ainda não foi gerado um número significativo de documentos, pelo menos no âmbito da produção brasileira no assunto.

Até o momento, quatro revisões de literatura sobre marketing no setor de informação (Freeman \& Katz, 1978; Tucci, 1988; Arnold, 1990; Webber, 1998) foram realizadas em âmbito internacional, divulgadas no Annual Review of Information Science and Technology (ARIST).

Outro aspecto interessante é a dificuldade em recuperar documentos sobre assuntos específicos na área de marketing em unidades de informação, a partir da consulta às fontes bibliográficas consideradas como as mais importantes para a pesquisa bibliográfica em Ciência da Informação e Biblioteconomia: o Library and Information Science Abstracts (LISA) e o Library Literature. Nestas obras, a terminologia específica de marketing não é considerada em nível de precisão adequado ao detalhamento de assunto na abordagem mercadológica.

Daí a curiosidade em pesquisar, como exemplo esclarecedor para esta situação, um tópico sobre a aplicação dos conceitos mercadológicos em unidades de informação, verificando o que foi considerado na área de informação a respeito desse tópico. Foi escolhida, então, a clássica abordagem dos $4 \mathrm{Ps}$ do composto de marketing.

Os 4Ps são a denominação preconizada pelo professor Jerome Mc Carthy (1978), conhecida maneira de estudar produto, preço, praça e promoção como componentes do composto de marketing.

O resultado da busca bibliográfica, identificando os trabalhos que abordassem a utilização dos 4Ps em unidades de informação, seria a fonte indicada para encontrar respostas às questões relativas à evolução da transferência dos conceitos mercadológicos para a Ciência da Informação, no que se refere ao enfoque dos 4Ps em unidades de informação.

Nessa perspectiva, o objetivo deste estudo foi analisar nessa literatura específica, durante o período de 1975 a 1995, que autores escreveram sobre o assunto, respondendo às seguintes questões:

- Quantos autores escreveram sobre os 4Ps?

- Qual o autor mais produtivo entre eles?
- Quem foi o pioneiro a escrever sobre os 4Ps em unidades de informação?

- Qual o pioneiro a propor adição de Ps?

- Quantos autores propuseram e adição de Ps aos 4Ps?

- Qual a concepção mais freqüente entre os fundamentos das propostas apresentadas?

- Qual a proposta mais completa de acordo com os critérios de observação da análise realizada para esse estudo?

Por tratar-se de assunto específico da área de marketing, cuja adoção pelo setor de informação não está totalmente disseminada, alguns pontos serão abordados, visando o entendimento dos 4Ps.

\section{ENTENDENDO OS 4 PS DO COMPOSTO MERCADOLÓGICO}

O composto mercadológico ou composto de marketing é conhecido também como marketing mix, definido por Kotler (1998, p. 97) como "o conjunto de ferramentas que a empresa usa para atingir seus objetivos de marketing no mercado-alvo."

Para Mc Carthy (1978), mercado-alvo é "o mais homogêneo ou similar grupo de consumidores para os quais a empresa deseja chamar atenção." Esse autor defende que a estratégia de marketing especifica o mercado-alvo e seu relacionado marketing mix. Na sua opinião, o consumidor é o centro do marketing mix, que inclui "algum produto, oferecido a um preço, com alguma promoção para contar aos potenciais consumidores sobre o produto e o modo de alcançar essa praça de consumidores". Para o autor, o consumidor é colocado no centro do diagrama do composto de marketing, mas não faz parte dele. O consumidor deve ser o alvo de todos os esforços de marketing e por isso ele é visto no centro do diagrama, representando os consumidores específicos que integram o mercadoalvo.

A história do composto de marketing se inicia com a aplicação das técnicas mercadológicas no setor lucrativo, quando, aproximadamente em 1950, inspirado nas idéias de Culligton, Neil N. Borden (1965) começou a usar o termo marketing mix em suas aulas e textos.

Para Borden (1965), a lista dos elementos do marketing mix poderia ser grande ou pequena, dependendo de como fosse usada uma classificação ou sub-classificação dos procedimentos e políticas mercadológicos com os quais a administração de marketing se envolvesse. No começo dos seus estudos, 
ele apresentou vários elementos do marketing mix. Mais tarde, ele mesmo abreviou sua lista de elementos iniciais, em função de sua própria percepção das questões e problemas relacionados com o marketing propriamente dito.

Entretanto, foi em 1960, que Jerome E. McCarthy (1978), na primeira edição do seu livro Basic Marketing: a managerial approach, apresentou uma estrutura inovadora, usando a classificação do marketing mix, que ele chamou de 4Ps, popularizando produto, praça, promoção e preço, como os seus quatro elementos.

Segundo Silveira (1992, p. 31), em 1988, Kotler propôs novos 4Ps, substituindo os propostos por Jerome McCarthy em 1960. Apresentados na língua inglesa, os novos 4Ps propostos por Kotler não guardam as mesmas letras iniciais, quando traduzidos para o português e, por essa razão, são apresentados a seguir no idioma original:

1.Probing, valorizando a pesquisa para entender o mercado consumidor;

2.Partitioning, enfocando a segmentação para fragmentar o mercado em grupos cada vez menores de consumidores;

3.Prioritizing, destacando a prioridade para seleção dos grupos de consumidores de interesse estratégico para a organização;

4. Positioning, ressaltando o posicionamento para desenvolver uma estratégia diferenciada e competitiva para cada um dos segmentos do mercado da organização.

Com base na proposta de Kotler (1988), Silveira (1994, p. 32) alertou que na década de 90, os novos acontecimentos tecnológicos, sociais, econômicos e políticos, entre outros afetariam aqueles que elegessem o conceito de marketing como estilo de gestão, pois essa escolha implicaria na revisão dos meios ou canais e elementos de marketing.

$\mathrm{Na}$ opinião de Lauterborn apud Schultz et alii (1994, p. 14), a teoria dos 4Ps foi válida para o seu tempo e cultura, mas a fórmula funcionou de cima para baixo na empresa. Foi imposta pela cúpula e estava orientada mais para os produtos do que para os consumidores. O fabricante decidia fazer um produto, porque poderia estabelecer seu preço para cobrir os custos e obter lucro tanto quanto possível, colocá-los nas prateleiras das lojas através da rede de distribuição e promovê-lo, desavergonhadamente. Por essas razões, propõe os $4 \mathrm{Cs}$, em substituição à teoria de McCarthy. Ele recomenda o uso de Cliente, em lugar de Produto; Custo possível para o cliente, em substituição ao Preço; Conveniência no lugar do Ponto de Distribuição e Comunicação, substituindo a Promoção. Lauterborn sugere:
1.Esqueça o Produto: estude as vontades e as necessidades do consumidor ou Cliente. Você não pode mais vender tudo o que consegue fabricar, pode apenas vender o que alguém especificamente quer adquirir.

2. Esqueça o Preço: compreenda o Custo para o consumidor satisfazer sua vontade ou necessidade.

3. Esqueça o Ponto de Distribuição (Praça) e pense na Conveniência de comprar.

4.Esqueça a Promoção, pois a palavra de ordem da década de 90 é a Comunicação.

Diante dessa realidade específica do setor lucrativo e considerando a importância da adoção do marketing em unidades de informação, observa-se que muitos aspectos precisam ser entendidos e disto dependerá essencialmente a transposição dos conceitos mercadológicos para essas organizações. Assim é oportuno conhecer o quê já foi estudado e abordado na literatura de Ciência da Informação sobre marketing e a proposta inicial é apreciar a abordagem dos $4 \mathrm{Ps}$ em unidades de informação.

\section{PROCEDIMENTOS METODOLÓGICOS DA PESQUISA}

Inicialmente, foram consultados os dois índices mais importantes para a pesquisa bibliográfica em Ciência da Informação e Biblioteconomia: o Library and Information Science Abstracts (LISA) e o Library Literature. A busca bibliográfica sobre os 4Ps em unidades de informação considerou a literatura indexada no período de 1975 a 1995.

Como se esperava, para atingir o objetivo da pesquisa, foi necessário o exame e leitura dos itens recuperados no levantamento realizado, pois a abordagem específica sobre os 4Ps não estava incluída nos termos usados na indexação das fontes consultadas. Além disso, apenas a leitura dos resumos dos documentos recuperados não foi suficiente, pois foi observado que às vezes o resumo mencionava o composto de marketing, mas o documento não abordava o tema, especificamente. Outras vezes, embora o assunto não fosse mencionado no resumo, o documento apresentava esse enfoque, mais detalhadamente. Esta condição limitou o estudo à apreciação apenas de documentos cujo acesso fosse possível no momento da realização da pesquisa na Universidade de Wisconsin, Madison, Estados Unidos, em fevereiro de 1996.

Além dos artigos de periódicos recuperados na busca aos índices, foram considerados outros 
documentos disponíveis, tais como verbetes de enciclopédias, capítulos de livros e anais de eventos sobre marketing em unidades de informação também foram analisados, apesar de não ser possível assegurar a exaustividade dos documentos produzidos sobre o assunto em tela na análise realizada. Em virtude da necessidade de acesso ao documento primário, não foi analisada a literatura brasileira, uma vez que os periódicos em Português não integravam o acervo das bibliotecas consultadas e não foram obtidas as cópias em tempo hábil para inclusão dos dados na pesquisa.

Foi considerado um total de 60 documentos, incluindo 37 artigos e 23 de outras categorias, entre capítulos de livros, verbetes de enciclopédias e trabalhos apresentados em eventos.

\section{RESULTADOS OBTIDOS}

A análise dos documentos obtidos permitiu a observação de interessantes pontos, destacados a seguir na tabela que os apresenta em ordem cronológica crescente por data de publicação, indicando o tipo de documento $(\mathrm{A}=$ artigo, $\mathrm{C}=$ capítulos de livros e outros tipos de documentos), se o autor denomina de 4Ps o composto mercadológico e se apresenta proposta acrescentando Ps.

A seguir, são apresentadas as respostas das questões da pesquisa.

\subsection{Quantos autores escreveram sobre os 4Ps em unidades de informação?}

Escreveram sobre a aplicação dos 4Ps em unidades de informação 47 autores, figurando entre eles nove autorias em parceria.

\subsection{Qual o autor mais produtivo sobre essa abordagem?}

A autora mais produtiva foi Darlene E. Weingand, que escreveu dez documentos, sendo três artigos de periódicos e sete capítulos de livros, dos quais um integra um livro sobre marketing de sua autoria.

Entre os autores mais produtivos destacam-se Cronin (1984; 1985), como autor de três artigos de periódicos; Virgo (1984), que produziu dois trabalhos apresentados em eventos e a dupla Galvin \& Keiser (1994), que escreveu um livro e um artigo, apesar dos nomes das autoras aparecerem em ordens diferentes nas publicações (Galvin \& Keiser, 1994 e Keiser \& Galvin, 1995).

Os demais 44 autores, que produziram sobre o tema, incluindo oito autorias em parceria, escreveram cada um deles apenas um trabalho, comprovando a lei de Lotka sobre a produtividade, uma vez que poucos autores $(n=4)$ produziram um número maior de trabalhos e muitos $(n=44)$ produziram somente um documento.

$\mathrm{O}$ ano mais produtivo do período analisado foi o de 1984, apontando significativa contribuição de Weingand (1984), uma vez que além de ter escrito dez documentos, Weingand foi responsável pela edição dos anais de um evento sobre marketing, que divulgou dois trabalhos de Virgo (1984) e um de Leisner (1984) sobre os 4Ps.

\subsection{Qual o autor pioneiro a escrever sobre os 4Ps em unidades de informação?}

Em 1981, Moulton (1981) foi o primeiro autor de artigo de periódico a abordar o tema. Antes dele, o tópico foi abordado por Bellardo \& Waldhart (1977), em 1977, que comentaram cada um dos elementos, mas não utilizaram os termos praça e promoção, optando pelo uso de comunicação e distribuição. Dragon, em 1979, e Edinger em 1980, também abordaram o assunto, mas não mencionaram os 4Ps para denominar os elementos do marketing mix.

Em 1976, Weinstock (1976) incluiu um tópico sobre os serviços de informação em Ciência e Tecnologia na Encyclopedia of Library and Information Science. Ele considerou produto, praça, promoção e preço como elementos básicos para proporcionar as respostas às pesquisas mercadológicas, mas não denominou esses elementos como os 4Ps.

\subsection{Qual o autor pioneiro a propor adição de Ps ao composto de marketing para unidades de informação?}

Quanto à determinação do autor pioneiro a apresentar proposta acrescentando Ps, o fato dependerá do ponto de vista em que a análise dos trabalhos for considerada. Isto porque:

a) Darlene Weingand (1986) pode ser considerada a pioneira, se considerarmos que o artigo escrito por ela foi publicado em outubro de 1986;

b) por outro lado, no mesmo ano foi publicado o artigo de Fruitholf (1986), no primeiro 
Tabela 1: Cronologia dos documentos analisados

\begin{tabular}{|c|c|c|c|c|}
\hline AUTOR & DATA & TIPO & DENOMINA 4PS? & ACRESCENTA Ps? \\
\hline Weinstock & 1976 & $\mathrm{C}$ & NÃO & NÃO \\
\hline Bellardo \& Waldhart & 1977 & A & NÃO & NÃO \\
\hline Dragon & 1979 & A & NÃO & NÃO \\
\hline Edinger & 1980 & A & NÃO & $\mathrm{NÃO}$ \\
\hline Moulton & 1981 & A & SIM & NÃO \\
\hline Condous & 1983 & A & NÃO & NÃO \\
\hline Weingand & 1983 & A & SIM & NÃO \\
\hline Cronin & 1984 & A & NÃO & NÃO \\
\hline Eisner, Joseph & 1984 & $\mathrm{C}$ & SIM & NÃO \\
\hline Mathews & 1984 & $\mathrm{C}$ & NÃO & NÃO \\
\hline Simpson & 1984 & $\mathrm{C}$ & SIM & NÃO \\
\hline Virgo (A marketing approach...) & 1984 & $\mathrm{C}$ & SIM & NÃO \\
\hline Virgo (The marketing audit...) & 1984 & $\mathrm{C}$ & SIM & NÃO \\
\hline Weingand (Marketin: its...) & 1984 & $\mathrm{C}$ & NÃO & NÃO \\
\hline Weingand (The application) & 1984 & $\mathrm{C}$ & NÃO & NÃO \\
\hline Weingand (Marketing with a smile) & 1984 & $\mathrm{C}$ & SIM & NÃO \\
\hline Wood, Douglas & 1984 & A & SIM & NÃO \\
\hline Bell & 1985 & $\mathrm{C}$ & NÃO & NÃO \\
\hline Cronin (Improving your ...) & 1985 & A & SIM & NÃO \\
\hline Cronin (Information services ...) & 1985 & A & SIM & NÃO \\
\hline Freeman \& O'Connell & 1985 & $\mathrm{C}$ & SIM & NÃO \\
\hline Fruitholf & 1986 & A & SIM & SIM \\
\hline Leisner & 1986 & A & SIM & SIM \\
\hline Warnken & 1986 & $\mathrm{C}$ & SIM & SIM \\
\hline Weingand & 1986 & $\mathrm{~A}$ & SIM & SIM \\
\hline Wood, Elizabeth & 1986 & $\mathrm{C}$ & SIM & NÃO \\
\hline Weingand & 1987 & $\mathrm{C}$ & SIM & SIM \\
\hline Char & 1988 & A & SIM & NÃO \\
\hline Montouri & 1988 & $\mathrm{~A}$ & NÃO & SIM \\
\hline Strauss & 1988 & A & NÃO & NÃO \\
\hline Dalton & 1989 & A & SIM & NĀO \\
\hline Norman & 1989 & $\mathrm{~A}$ & SIM & NÃO \\
\hline Dean & 1990 & $\mathrm{C}$ & NÃO & NÃO \\
\hline Eisner, Joe & 1990 & A & SIM & $?$ \\
\hline Kinnell & 1990 & A & SIM & NÃO \\
\hline Lett & 1990 & $\mathrm{C}$ & SIM & NÃO \\
\hline Wright \& Nicholson & 1990 & $\mathrm{C}$ & SIM & NÃO \\
\hline Cooper & 1991 & A & SIM & NÃO \\
\hline Gallacher & 1991 & $\mathrm{C}$ & NÃO & NÃO \\
\hline Weingand & 1991 & $\mathrm{C}$ & SIM & NÃO \\
\hline Yates-Mercer \& Steward & 1991 & A & SIM & NÃO \\
\hline Amaral & 1991 & A & NĀO & NÃO \\
\hline Simpock-Vieweg & 1992 & A & NÃO & NÃO \\
\hline Collins \& Glyptis & 1992 & A & SIM & NÃO \\
\hline Kinnell \& McDougall & 1992 & A & SIM & NÃO \\
\hline Dijkstra & 1992 & A & NÃO & NÃO \\
\hline McCarthy & 1992 & $\mathrm{~A}$ & SIM & NÃO \\
\hline Morgan \& Noble & 1992 & A & NÃO & NÃO \\
\hline Smith \& Saker & 1992 & A & SIM & SIM \\
\hline Walters & 1992 & $\mathrm{C}$ & SIM & NÃO \\
\hline Weingand & 1992 & $\mathrm{C}$ & SIM & NÃO \\
\hline Booth & 1993 & A & SIM & NÃO \\
\hline Lindquist & 1993 & A & NÃO & SIM \\
\hline Galvin \& Keiser & 1994 & A & NÃO & NÃO \\
\hline Rowley & 1994 & A & SIM & SIM \\
\hline Weingand & 1994 & $\mathrm{C}$ & SIM & NÃO \\
\hline Dimick & 1995 & A & SIM & NÃO \\
\hline Keiser \& Galvin & 1995 & $\mathrm{C}$ & SIM & NÃO \\
\hline Powers & 1995 & $\mathrm{~A}$ & SIM & SIM \\
\hline Weingand & 1995 & A & SIM & NÃO \\
\hline
\end{tabular}


fascículo de um título de periódico publicado em 1986, que não se refere ao mês de publicação. Outro artigo foi escrito por Leisner (1986) no mesmo ano, embora a data do periódico reporte-se ao outono, que vai de outubro a dezembro, no país onde o periódico foi publicado. Deste modo, se for considerado somente o ano, os três autores podem ser considerados pioneiros.

\subsection{Quantos autores propuseram a adição de Ps aos 4Ps?}

Um total de nove autores apresentaram propostas para adicionar Ps. Entre eles, uma autoria era em parceria dupla, e Weingand (1986 e 1987) apresentou sua proposta inicialmente sob a forma de artigo e depois incluiu-a no seu livro.

\subsection{Qual a concepção mais freqüente entre os fundamentos das propostas apresentadas?}

A análise das propostas apresentadas revelou que embora os dois artigos escritos por Cronin (1985 e 1985) não tivessem apresentado propostas, acrescentando elementos aos 4Ps, o autor questionou a adoção dos 4Ps em unidades de informação, admitindo que os livros-texto sobre marketing faziam referência aos 4Ps, mas era necessário assegurar uma apropriação social dessa abordagem pelas unidades de informação.

As propostas apresentadas por Rowley (1984), Smith \& Saker (1992) e Fruitholf (1988) sugeriram o acréscimo de elementos conforme propostas de outros autores do setor lucrativo:

c) Rowley sugeriu a adição de Processamento, referindo-se à coleção de dados, análise dos dados e apresentação de dados, conforme proposta apresentada inicialmente por Beaumont;

d) Smith \& Saker, baseados em Booms \& Bitner, enfatizaram o fato de os serviços serem providenciados por Pessoas e daí a qualidade da equipe tornar-se essencial para determinar a qualidade da serviço oferecido, sendo recrutamento e treinamento elementos vitais na provisão desses serviços. Da mesma forma, é reforçada a importância do Processo ou sistema utilizado pela equipe, considerado outro $\mathrm{P}$ adicional aos $4 \mathrm{Ps}$, juntamente com o elemento final da proposta, em inglês physical evidence, para justificar outro $\mathrm{P}$, cuja tradução para o Português descaracteriza-se o P, significando a própria prestação do serviço ao consumidor que lhe permite o julgamento da qualidade de serviço prestado;

e) Fruitholf apresenta sua proposta fundamentada na proposta de Bosman, que ressalta a relevância dos Participantes, considerando os empregados da empresa e os consumidores; Physical evidence, como o ambiente físico do serviço e o Processo, incluindo todos os aspectos dos procedimentos empregados na prestação dos serviços;

f) Leisner (1988), Warnken (1986), Weingand (1986 e 1987), Montouri (1988), Lindquist (1993) e Powers (1995) apresentaram suas próprias propostas para acrescentar Ps.

Três propostas (Montouri, 1988, Lindquist, 1993 e Powers, 1995) não denominaram a classificação do marketing mix como os 4Ps:

g) Lindquist, em seu pequeno artigo publicado em 1993, sugeriu a inclusão do termo Pessoas, mas não explicou sua sugestão em detalhes, limitando-se a considerar organização, competência e capacidade como variáveis de marketing;

h) o artigo de Montouri, publicado em 1988, ressaltou a importância dos Profissionais como os bibliotecários e a equipe de trabalho como o suporte indispensável para o esforço de marketing, mas não incluiu em seu trabalho explicações sobre a sugestão, nem mencionou os 4Ps, referindo-se ao marketing mix;

i) o mesmo ocorreu com o artigo de Powers, em 1995, quando o autor externou sua opinião sobre os Ps de marketing, incluindo Público, ao referir-se aos clientes que usam a biblioteca, enfatizando que conhecer a demanda do produto é essencial para o sucesso desse produto.

Entre as sete propostas que denominaram o marketing mix como os 4Ps, a proposta de Rowley (1994) foi a única que não apresentou comentários sobre cada um dos elementos.

Entre os sete autores que propuseram acréscimo de Ps, as propostas de Warnken (1986), Montouri (1988), Lindquist (1993) e Powers (1995) fundamentaram-se em aspectos relacionados à importância dos recursos humanos e a proposta de Leisner (1986) mencionou Política pública como sugestões a serem acrescentadas 
e apreciadas no estudo do marketing mix. Nenhuma proposta focalizou especificamente os consumidores. Ao apresentar sua proposta, Warnken (1986) concentrou sua preocupação na Participação, considerando-a como o elemento inseparável dos componentes do marketing mix.

\subsection{Qual a proposta mais completa de acordo com os critérios de análise desse estudo?}

Como critério de avaliação das propostas foi observado se a mesma incluía fundamentação ou esclarecimentos sobre a sua aplicação prática, ou se apenas era mencionada como sugestão.

A maior parte das propostas apresentadas não incluiu informação suficiente para esclarecer sua sugestão em âmbito de uma aplicação prática, que facilitasse o entendimento da própria proposição. Por essa razão, a proposta de Weingand (1986 e 1887) foi considerada a mais completa. A autora apresentou sua abordagem primeiramente sob a forma de artigo e, mais tarde, incluiu-a em um dos capítulos de seu livro sobre marketing. Ela propôs os termos Prelúdio e Poslúdio, compondo sua visão de seis elementos para o estudo do marketing mix. Ela destaca que vivemos em um ambiente de constantes mudanças que precisam ser admitidas e estudadas na fase da auditoria de marketing, que ela denomina de prelúdio. Reforça também a importância da avaliação a realizar para verificar os resultados dos esforços de marketing, na fase denominada por ela de poslúdio. A proposta pode ser considerada completa e detalhada, pois apresenta todas as informações necessárias para ser entendida pelos que desejarem aplicá-la na prática.

\section{CONSIDERAÇÕES FINAIS}

Apesar das limitações do estudo, foi possível apreciar a literatura de um tema específico sobre o adoção das técnicas de marketing em unidades de informação, observando formas diversas na interpretação de aspectos conceituais, de acordo com o ponto de vista de diferentes autores. As dificuldades em recuperar trabalhos sobre abordagens específicas de marketing na literatura de Ciência da Informação, a partir dos principais índices bibliográficos da área, foi significativa na ocasião em que este estudo foi realizado (fevereiro de 1996). Acredita-se que possam ser usados, como alternativa para superar estas dificuldades, mecanismos de busca mais sofisticados. A recuperação da informação disponível na Internet, apesar de toda sua anarquia, tem evoluído bastante. Entretanto, embora atenuados, não são eliminados, totalmente, os problemas relativos à terminologia. Essa problemática terminológica é muito mais complexa quando se trata da indexação utilizada nos índices.

Sugere-se que a terminologia específica de marketing da informação seja alvo de pesquisa a ser desenvolvida em parceria entre estudiosos das áreas de terminologia e de marketing em unidades de informação. Tal providência poderá ser uma significativa contribuição para o avanço da transferência dos conceitos mercadológicos para o setor da informação, facilitando a recuperação de documentos produzidos sobre o tema.

Assim, seriam facilitadas as condições de pesquisa para o entendimento da filosofia de marketing no processo de transferência de princípios mercadológicos para aplicá-los no planejamento e tomada de decisão gerencial em unidades de informação. Sob esse aspecto, destaca-se a opinião de Weingand (1987, p. 17), quando afirma: marketing sem planejamento é um exercício e planejamento sem marketing é uma formalidade.

Os trabalhos analisados sobre os 4Ps são importantes para auxiliar os profissionais interessados em adotar técnicas mercadológicas em unidades de informação. Podem representar o destaque de importantes tópicos para reflexão. Entretanto, além de entender o assunto, os profissionais da informação precisam romper as quatro paredes do local de trabalho, buscando encontrar a fundamentação necessária ao desenvolvimento das atividades de marketing, aprendendo que as unidades de informação devem ser vistas como um "negócio" igual aos demais, embora guardadas suas especificidades.

\section{REFERÊNCIAS BIBLIOGRÁFICAS}

AMARAL, Sueli Angelica do. Marketing adoption in Brazilian Geoscience and Mineral Technology libraries: a study of library managers' opinions. Infomediary, v. 5, n. 2/3, p. 95-104, 1991

ARNOLD, Stephen E. Marketing eletronic information: theory, practice and challenges. Annual Review of Information Science and Technology, v. 25, p. 87-4, 1990.

BELL, Jo Ann. Marketing reference services: translating selected concepts into action for a specific practice setting. In: WOOD, M. Sandra, ed. Cost analysis, cost recovery, marketing, and fee-based services: a guide for the Healthy Science librarian. New York: The Haworth Press, 1985. p. 127-142. 
BELLARDO, Trudi; WALDHART, Thomas J. Marketing products and services. Libri, v. 27, n. 3, p. 181-194, Mar. 1977.

BOOTH, Jennifer. Marketing public library services: the gap between theory and reality. Library Management, v. 14, n. 1, p. 9-23, 1993.

BORDEN, N. h, The concept of the marketing mix. In: SCHWARTZ, G. Science in marketing. Wiley, Chichester, 1965. p. 386-397.

CHAR, Lim Kim. Library marketing and its application to public libraries. Singapore Libraies, v. 18, p. 19-33, 1988.

COLLINS, Michel F.; GLYPTIS, Susan A. Marketing public leisure services in the UK. Library Management, v. 13, n. 4, p.33-42,1992.

CONDOUS, Crystal. Non-profit marketing- libraries' future? Aslib Proceedings, v.35, n.10, p.407-417, Oct.1983.

COOPER, Sue. Marketing strategies for growth in special libraries I. New Zealand Libraries, v. 46, n.9, p.16-19, Mar., 1991.

CRONIN, Blaise. Improving your I.Q. innovation quotient. Information and Libray Manager, v.5, n.3, p.25-32, Dec. 1985

CRONIN, Blaise. Information services marketing, v. 53, n.3, p.115-119, Sept. 1985.

CRONIN, Blaise. The marketing of public library services in the UK; practical applications. The European Journal of Marketing, v.18, n.2, p.45-55, Oct.1984.

DALTON, Gwenda M. E. Marketing communication: a means to achieve a positive professional image. South African Journal of Library and Information Science, v. 57, n.2, p.184-190,1989.

DEAN, Sharon. Part 1: definitions. In: DEAN, Sharon. Winning marketing techniques: an introduction to marketing for information professionals, a self study program. Washington: Special Libraries Association,1990. p.7-23.

DIJKSTRA, Jan Willem. Factors in setting prices of journals. Collection Management, v.15, n. 3/4, p.533-540,1992.

DIMICK, Barbara. Marketing youth services. Library Trends, v.43, n.3, p.463-477, Winter 1995.

DRAGON, Andrea C. Marketing the library. Wilson Library Bulletin, v.53, n.7, p.498-502, Mar.1979.

EDINGER, Joyce A. Marketing library services: strategy for survival. College \& Research Library, v.41. n.4, p.328-332, July 1980 .

EISNER, Joe. The marketing budget: how much is enough? The Bottom Line, v.4, n.2, p.33-34, Summer 1990.

EISNER, Joseph. The fallacy of applying traditional market pricing theory to tax-supported public libraries. In: WEINGAND, Darlene E., ed. Marketing for libraries and information agencies. Nonwood, New Jersey: Ablex Publishing Corporation,1984. p.29-33.
FREEMAN, Joan K.; O'CONNELL, Kathie A. The marketing of biomedical information service at the University of Minnesota: creative error correction. In: WOOD, M. Sandra, ed. Cost analysis, cost recovery, marketing, and fee-based services: a guide for the Healthy Science librarian. New York: The Haworth Press,1985. p.159-171.

FREEMAN, James E.; KATZ, Ruth M. Information marketing. Annual Review of Information Science and Technology, v.13, p.37-59,1978.

FRUITHOLF, A. C. Marketing in public library services in Belgium. Mousaion, v.4, n.1, p.38-64,1986.

GALLACHER, Margaret. Marketing techniques applied to the development and presentation of a seminar programme. In: WOOLS, Blanche, ed. Continuing professional education: an IFLA Guidebook. A publication of the Continuing Professional Education Round Table (CPERT) of the International Federation of Library Associations and Institutions. Munchen: K.G.Saur, 1991. (IFLA Publications, 55) p.58-60.

GALVIN, Carol K.; KEISER, Barbie E. A market-driven approach for the library/information center. FID News Bulletin, v.44, n.1, p. 5-11, Jan.1994.

KEISER, Barbie E.; GALVIN, Carol K. The marketing mix. In: KEISER, Barbie E., GALVIN, Carol K. Marketing library services: a nuts-and-bolts approach. 4 th ed. The Hague: FID, 1995. (FID Occasional Paper, n.9). p.49-68.

KINNELL, Margaret. Marketing first, promotion later: a review. Outlook on Research Libraries, p.3-6, Nov. 1990.

KINNELL, Margaret; MACDOUGALL, Jennifer. Libraries and leisure services marketing: rhetoric and reality. Public Library Journal, v.7, n.4, p.93-97, July/ Aug.1992.

KOTLER, Philip. Administração de marketing: análise, planejamento, implementação e controle. $5^{\mathfrak{a}}$ ed. São Paulo: Atlas, 1998. 725p.

KOTLER, Phillip; LEVY, S. J. Broadening the concept of marketing. Journal of Marketing, v.33, n.1, p.10-15, Jan. 1969 .

LEISNER, Tony. Mission statements and the marketing mix. Public Libraries, v.25, p.86-87, Fall 1986.

LETT, Brenda. Training for marketing and public relations in libraries. In: PRYTHERCH, Roy, ed. Handbook of library training practice. Gower, 1990. p.49-71.

LINDQUIST, Mats G. The marketing of information services. FID News Bulletin, v.43, n.11/12, p.267-269, Nov./ Dec. 1993.

McCARTHY, E. Jerome. Basic marketing: a managerial approach. 6th ed. Richard D. Irwin, Homewood, 1978.

McCARTHY, Grace. Promoting the in-house library. Aslib Proceedings, v. 44, n.7/8, p.289-293, July/Aug. 1992. 
MATHEWS, Anne J. The use of marketing principles in library planning. In: WEINGAND, Darlene E., ed. Marketing for libraries and information agencies. Nonwood, New Jersey: Ablex Publishing Corporation, 1984. p.3-14.

MONTOURI, Charles F. Marketing and public libraries: the commitment. North Carolina Libraries, v.46, n.3, p.148-150,152-153, Fall 1988.

MORGAN, Phillip; NOBLE, Sue. Marketing library and information services in the ' 90 s. The Australian Library Journal, v.41, n.4, p.283-292, Nov.1992.

MOULTON, Bethe. Marketing and library cooperatives. Wilson Library Bulletin, v.55, n.5, p.347-352, Jan.1981.

NORMAN, O. Gene. Marketing library and information services: an annotated guide to recent trends and developments. Reference Services Review, v.17, n.1, p.43-64, Spring 1989.

POWERS, Janet E. Marketing in the special library environment. Library Trends, v.43, n.3, p.478-493, Winter 1995.

ROWLEY, Jennifer. Marketing information systems. Aslib Proceedings, v.46, n.7/8, p.185-187, July/Aug.1994.

SCHULTZ, Don E.; TANNENBAUM, Stanley I.; LAUTERBORN, Robert F. O novo paradigma do marketing: como obter resultados mensuráveis através do uso do database e das comunicações integradas do marketing. São Paulo: Makron Books, 1994.

SILVEIRA, Amélia. Marketing directo; proposición de modelo para los servicios de información. Florianópolis: Biblioteca Universitária da UFSC,1992.

SIMPOCK-VIEWEG, Kathy. How to develop a marketing plan for a firm library. Law Library Journal, v.84, n. 1, p.67-91, Winter 1992.

SIMPSON, Thomas. The marketing challenge in public library. In: WEINGAND, Darlene E., ed. Marketing for libraries and information agencies. Nonwood, New Jersey: Ablex Publishing Corporation,1984, p.21-28.

SMITH, Gareth; SAKER, Jim. Developing marketing strategy in the not-for-profit sector. Library Management, v.13, n.4, p.6-21,1992.

STRAUSS, Diane. Marketing fundamentals for librarians. North Carolina Libraries, v.46, n.3, p.132-135, Fall 1988.

TUCCI, Valerie K. Information marketing for libraries. Annual review of Information Science and Technology, v. 23, p. $59-82,1988$.

VIRGO, Julie A. C. A marketing approach to identifying library services. In: MARKETING public library services: new strategies. Proceedings of Public Library Association Frontiers Conference. Madison, WI, 1984. Compiled by Darlene E. Weingand. Madison: American Library Association,1984. p.17-27.
VIRGO, Julie A. C. The marketing audit in the public library. In: MARKETING public library services; new strategies. Proceedings of Public Library Association Frontiers Conference. Madison, WI,1984. Compiled by Darlene E. Weingand. Madison: American Library Association, 1984. p.33-45.

WALTERS, Suzanne. Marketing strategies. In: WALTERS, Suzanne. Marketing: a how-to-do-it manual for librarians. New York: Neal-Schuman Publishers, Inc., 1992. p.37-49.

WARNKEN, Paula. Design and promoting the "right" BI program: a holistic approach. In: KIRKENDALL, Carolyn A., ed. Marketing instructional services: applying private sector techniques to plan and promote bibliographic instructions. Papers presented at the 13 th Library Instruction Conference held at Eastern Michigan University, May 3-4,1984. Ann Arbor: Pierian Press, 1986. p.55-63.

WEBBER Sheila Anne Elizabeth. Pricing and marketing online information services. Annual Review of Information Science and Technology, v.33, p.39-83, 1998.

WEINGAND, Darlene E. The application. In: WEINGAND, Darlene E., ed. Marketing for libraries and information agencies. Nonwood, New Jersey: Ablex publishing Corporation, 1984. p.99.

WEINGAND, Darlene E. Distribution of the library's product: the need for innovation. Journal of Library Administration, v.4, n.4, p.49-57, Winter 1983.

WEINGAND, Darlene E. Marketing and financial management keys to fiscal accountability in the public library. In: KEEPING the books. Fort Atkinson: Highsmith Press,1992. p.307-316.

WEINGAND, Darlene E. Marketing continuing professional education. In: WOOLS, Blanche, ed. Continuing professional education: an IFLA Guidebook. A publication of the Continuing Professional Education Round Table (CPERT) of the International Federation of Library Associations and Institutions. Munchen K.G.Saur,1991. (IFLA Publications, 55) p.52-57.

WEINGAND, Darlene E. Marketing: its relationship to the planning process. In: MARKETING public library services; new strategies. Proceedings of Public Library Association Frontiers Conference. Madison, WI, 1984. Compiled by Darlene E. Weingand. Madison: American Library Association,1984. p.28-30.

WEINGAND, Darlene E. Marketing: phase two. In: WEINGAND, Darlene E. Managing today's public library: blueprints for change. Englewood: Libraries Unlimited, Inc., 1994. p.132-148.

WEINGAND, Darlene E. Marketing with a smile. In: WEINGAND, Darlene E. Marketing/Planning library and information services. Littleton: Libraries Unlimited, Inc.,1987. p.3-10. 
WEINGAND, Darlene E. Preparing for the new millenium: the case for using marketing strategies. Library Trends, v.43, n.3, p.295-317, Winter 1995.

WEINSTOCK, M. Marketing science and technical information service. In: ENCYCLOPEDIA of Library and Information Science. New York: Marcel Dekker,1976. v.17, p.165-176.

WOOD. Douglas. Improving your image: how to promote a library or information Service. Aslib Proceedings, v.36, n.10, p.401-408, Oct.1984.

WOOD, Elizabeth J. Marketing for libraries: an introduction and overview. In: KIRKENDALL, Carolyn A. ed. Marketing instructional services: applying private sector techniques to plan and promote bibliographic instructions Papers presented at the 13th Library Instruction Conference held at Eastern Michigan University. May 3-4,1984. Ann Arbor: Pierian Press,1986, p.1-25.

WRIGHT, J.; NICHOLSON, F.. Marketing for libraries. In: ROCHESTER, M. K.; NICHOLSON, F.. ed. Challenges in Australian library management. Adelaide: Auslib Press, 1990. p.88-101

YATES-MERCER, Penelope A.; STEWARD, Yvonne F. The marketing of internal business information services. Journal of Information Science, v.17, n.4, p.221-233,1991. 